\title{
$\widehat{A}$ Madridge \\ madridge Journal of Nursing \\ interconnecting scientific World
}

Case Study

Open Access

\section{A Review and Case Study of Urinary Incontinence}

\author{
Emily Bagnola ${ }^{1}$, Edward Pearce ${ }^{1}$ and Barbara Broome ${ }^{2 *}$ \\ ${ }^{\prime}$ Honors Nursing Student, College of Nursing, Kent State University, Kent, Ohio, USA \\ ${ }^{2}$ Dean and Professor, College of Nursing, Kent State University, Kent, Ohio, USA
}

\section{Article Info}

\author{
*Corresponding author: \\ Barbara Broome \\ Professor of Nursing \\ Kent State University \\ USA \\ E-mail: Bbroome1@kent.edu
}

Received: April 04, 2017

Accepted: April 07, 2017

Published: April 12, 2017

Citation: Bagnola E, Pearce E, Broome B. A Review and Case Study of Urinary Incontinence. Madridge J Nurs. 2017; 2(1): 27-31.

doi: 10.18689/mjn-1000105

Copyright: (c) 2017 The Author(s). This work is licensed under a Creative Commons Attribution 4.0 International License, which permits unrestricted use, distribution, and reproduction in any medium, provided the original work is properly cited.

Published by Madridge Publishers

\begin{abstract}
Urinary incontinence $(\mathrm{UI})$, the involuntary leakage of urine due to the loss or weakened control of the urinary sphincter, is a crucial subject of medicine that has physiological, psychological, and sociological complications [25]. Urinary incontinence can range from mild leakage to an overflow amount of urine. Although this is a common problem affecting millions of Americans, it is not often a subject of discussion because it can be embarrassing for people to talk about, even with their physicians [2]. Physiologically unpleasant for patients, UI can also be emotionally demeaning, decreasing self-concept and perceived body image. UI can be divided into transient (acute) incontinence and chronic incontinence; they both have significant effects on the patient despite one lasting longer than the other. Causes of incontinence can originate from a variety of causes. Research indicates urinary incontinence may be related to genetic factors, biological aspects, and physiological characteristics [14]. Treatment for incontinence includes behavioral, pharmacological, and surgical interventions, as well as catheterization. Appropriate treatment is based the individual's current situation and follows evaluation of which treatment option will provide the best opportunity for the highest quality of life.
\end{abstract}

Keywords: Urinary incontinence; Self confidence; Genetic factors; Biological aspects; Physiological characteristics.

\section{Overview}

Urinary incontinence $(\mathrm{UI})$ is the involuntary leakage of urine. Urine is stored in the bladder, which is relaxed when a person is not urinating. When a person urinates, the bladder muscles constrict, thus forcing urine into the urethra, a tube that acts as a passageway to connect the urinary bladder and the external part of the body. During this process, the muscles around the urethra relax, allowing the urine to exit from the body. Urinary incontinence occurs when the muscles around the urethra relax involuntarily [3].

\section{Types}

Urinary incontinence can be classified into two categories: transient or chronic. Transient incontinence is a temporary, reversible, or acute type of incontinence. An illness or medical condition that is short-lived causes transient incontinence. Once the illness or condition is treated, the incontinence is treated by default. The other category of incontinence, chronic incontinence, consists of five different types: stress, urge, mixed, overflow, and functional [14]. Stress incontinence, the most common type of urinary incontinence in women, occurs when there is a loss of urine during physical activity or exertion such as coughing, sneezing, laughing, exercise, or any other movements which put pressure on the bladder [3,32]. Urge incontinence, often called overactive bladder, 
is the sudden need to urinate due to increased intraabdominal pressure or spasmodic activity of the bladder. A person with overactive bladder feels urgency and may leak before reaching the restroom. This commonly occurs while a person is sleeping, drinking water, looking at water, or touching water. When a person experiences symptoms of both stress and urge incontinence, it is referred to as mixed incontinence [7].When a person cannot completely empty their bladder while urinating, it is referred to as overflow incontinence. A common symptom experienced by a patient with this type of incontinence is "dribbling" after urination. Functional incontinence is a category of incontinence that is related to a mental or physical disability. While a patient might have a healthy urinary system, their disability impairs the ability to get to the restroom [37].

\section{Causes}

UI has a variety of systemic causes, including urinary dysfunction; neurological, musculoskeletal, and pharmacological issues; nutrition; genetics; and individual health. These areas of causation can produce symptoms that are both acute and chronic. Acute causes of urinary incontinence are generally observed to be short-term, and once these underlying conditions are treated, UI usually disappears. Within the urinary system, common causes of acute $\mathrm{UI}$ include prostate infection/inflammation, urinary tract infection (UTI), and stool impaction [19, 22, 24, 27, 29, 32]. An enlarged prostate or prostatic hypertrophy may cause infection and/or inflammation resulting in UI. The prostate is located just below the bladder and surrounds the urethra. Prostate enlargement can narrow the urethra, resulting in incomplete and delayed emptying which can result in a urinary tract infection (UTI).UTIs are infections of the bladder or the urethra. Although women are more prone to UTIs than men, UTIs are worrisome and related to infection in the bladder. UTIs cause the patient to feel the need to urinate more than usual or can make the individual feel as though he or she cannot empty their bladder completely [24].

Constipation may also cause UI. The rectum and the bladder share many of the same nerves [19]. When constipation occurs and there is bulk in the rectum from stool, the nerves can be over stimulated, resulting in an increased feeling of the need to urinate $[19,32]$. Other causes of UI are the shortterm nervous complications from alcohol or sedative drug withdrawal, drug abuse, electrolyte imbalances, infections, poisons, or surgery after which patients may experience mental confusion [12]. This type of confusion or delirium can result in situation-related UI [19,3]. Another common cause of $\mathrm{UI}$ is medications. Pharmacological agents, such as diuretics, antidepressants, cough and cold remedies, antihistamines, angiotensin converting enzyme (ACE) inhibitors, hormone replacement therapy, and sedatives can cause temporary incontinence because they alter the natural storing and passing of urine [24,32]. For example, in the renin-angiotensinaldosterone system in the genitourinary tract, ACE inhibitors prevent angiotensin I from being converted to angiotensin II, a vasoconstrictor which treats hypertension. ACE inhibitors also cause a decrease in urethral sphincter tone [8].

Nutrition and certain foods and drinks may mimic the effects of diuretic medications, including alcohol, caffeine, tea, coffee, carbonated drinks, artificial sweeteners, corn syrup, and foods high in spice, sugar, acid, and vitamins B and $\mathrm{C}$ [32].Nutritional factors can also change the $\mathrm{pH}$ of urine. The $\mathrm{pH}$ of urine ranges from 4.5-8 with the average $\mathrm{pH}$ being about 6, indicating that urine is slightly acidic [21]. An increase in consumption of certain vegetables, citrus fruits, and meat can lower the $\mathrm{pH}$ of urine, thus making it more acidic. Acidic urine can cause bladder spasms, leading to UI [11]. Lastly, the overall health of the individual can play a key role in acute UI, including the patient's weight and pregnancy status, which both cause increased intra-abdominal pressure [24].

As opposed to acute causes of urinary incontinence, chronic causes are not as easily reversible and have a longer duration. Chronic causes of UI are directly involved with the urinary system, including untreated benign prostate hyperplasia (BPH), bladder cancer, bladder fistula, bladder spasms, and bladder stones.BPH is an enlarged prostate. The prostate is a gland in men located between the penis and the bladder. If the prostate gland becomes enlarged, it can put pressure on the bladder. A tumor from bladder cancer can cause overflow incontinence if the tumor blocks the urethra. Often, the treatment of bladder cancer also lead to UI because radiation or surgery to remove the tumor can cause damage to the urethra. A bladder fistula is a connection formed between the vaginal wall and the bladder. The body forms a fistula when there is a block, typically from an obstruction during childbirth. UI is a consequence because urine bypasses the urethra and exits out the vagina. Bladder stones form when the urine becomes too concentrated with salts. These mineral stones can block the ureter, thus causing UI [24, 38].

Several neurological conditions, including Alzheimer's disease, depression, multiple sclerosis (MS), stroke, Parkinson's disease, and certain spinal cord injuries may cause chronic UI as well [24].Alzheimer's disease is a common form of dementia. Those who suffer from Alzheimer's lose mental functions such as memory and judgment. This loss of mental function can affect the ability to control the bladder. Depression and UI can go hand-in-hand. If a patient is depressed, he or she may be less likely to seek treatment for UI. Patients who experience UI may become depressed due to embarrassment.MS affects the nervous system. It can interfere with the nerve signals that help with the movement of urine. Therefore, urine might come out from the body before the patient is ready. A stroke can lead to muscle weakness, specifically around the bladder. Loss of muscle function around the bladder could cause UI. Parkinson's disease is a disorder that affects the nervous system. UI may result from Parkinson's because the brain does not communicate with the sphincter. As a result, the sphincter opens and releases urine when there is only a small amount. Damage to the spinal cord also disrupts communication from the brain to the sphincter [24].

From a musculoskeletal standpoint, connective tissue 
disorders and sphincter weakness can also affect continence. Connective tissue disorders can have long-term effects on UI because the connective tissue around the bladder is weaker [24]. Ehlers-Danlos syndrome is a connective tissue disorder that causes lax skin and hyper mobile joints. The organs, such as the bladder, can also become lax, and this condition may lead to the bladder dropping from its normal position and pushing against the vagina. This is known as pelvic prolapse, and UI can be a common side effect [31]. Spinabifida can cause sphincter weakness. Spinabifida is a condition in which the spinal cord does not develop properly, which may cause varying degrees of paralysis [9]. This can cause immobility, the leading factor for functional incontinence.

The nutritional intake of an individual can also have longlasting effects on urinary incontinence, such as persistent, poor fluid intake. If an individual is lacking proper fluid intake, his or her urine will eventually become concentrated. Concentrated urine irritates the bladder because it changes the $\mathrm{pH}$ of urine. The urine becomes more acidic, which leads to an over active bladder. Based on the individual, certain health and genetic factors play a significant role in urinary incontinence, such as age, menopause, and various birth defects [24].Ectopic ureter is a birth defect where the ureter does not terminate in the urinary bladder. Due to this structural abnormality, $\mathrm{UI}$ is a common consequence. Other types of birth defects include urethral duplication and ureterocele. Urethral duplication is when two ureters enter the bladder on the same side. This can cause the urine to back up into the kidneys.

Ureterocele is swelling at the bottom of the ureter which blocks the flow of urine. These birth defects may lead to UI due to the improper flow of urine [9].

\section{Treatment}

Much like the causes and risk factors, treatments of urinary incontinence can be categorized as behavioral, pharmacological, catheter, or surgical. The simplest example of a behavioral treatment is lifestyle change by the patient, such as monitoring consumption of fluids, quitting smoking, and losing weight. Another example of behavioral treatment is bladder training, where the goal is to increase the amount of time between emptying the bladder and the amount of fluid the bladder can contain. In order for bladder training to be successful, the patient must adhere to a strict voiding schedule, even if he or she does not feel the need to urinate. Another crucial aspect of bladder training is pelvic floor muscle exercises, commonly called Kegel exercises, which are non-evasive exercises that help strengthen the pelvic floor muscles. Pelvic floor muscles support vital organs that affect elimination, including the uterus or prostate, bladder, small intestine, and rectum. Men and women may both benefit from Kegel exercises.

The first step in Kegel exercises is for the patient to determine which muscles he or she needs to exercise in order to be effective. Stopping urination midstream helps to identify the muscles, but one common mistake that patients make is concentrating on the abdomen, thighs, and buttocks, which are not part of the pelvic floor muscle group. An instrument called a biofeedback mechanism can help a patient identify the correct muscles [20].According to the UCSF Medical Center (2017a), a biofeedback mechanism uses computer graphs and audible tones to show which muscles the patient is exercising and the strength of those muscles [36]. Once the patient has successfully identified which muscles are effective, they should perform these exercises about three times a day while standing, sitting and lying down and not during urination [11].The nurse's role in patient teaching is crucial, as the nurse will assist the patient in developing the skill to perform Kegel exercises. Kegel exercises are used to increase pelvic muscle strength to treat stress, urge, and mixed incontinence. Other strategies include "quick flicks" for urgency and bladder training. If the patient has to urinate before the scheduled time, he or she should use Kegel exercises to suppress the urge to urinate. Bladder training starts at small intervals and then can be increased to 30-minute increments once a goal is met. This continues until the patient is able to comfortably last for four hours without urinating [37].

Another common form of treatment is the use of pharmacologic agents, specifically anticholinergic medications. Anticholinergic drugs block acetylcholine (Ach), a neurotransmitter common in the parasympathetic nervous system. One common function of Ach is to send signals to the brain that trigger bladder contraction. Common types of anticholinergic medications prescribed for urinary incontinence include: Oxybutynin (Ditropan XL, Oxytrol), Tolterdoine (Detrol), Darifenacin (Enablex), Solifenacin (Vesicare), Trospium (Sanctura), and Fesoterodine (Toviaz).Another type of medication used for UI is Mirabegron (Myrbetriq), which relaxes the bladder muscle and can increase the amount of urine that the bladder can hold before having to urinate [18].Alpha-blockers and 5-alpha reductase inhibitors are common medications that are prescribed for men.Alphablockers, such as Terazosin (Hytrin), Doxazosin (Cardura), Tamsulosin (Flomax), Alfuzosin (Uroxatral), and Silodosin (Rapaflo), can help treat prostate enlargement and bladder obstruction complications by relaxing the smooth muscle in the prostate and bladder [26].

As reported by the National Institute of Diabetes and Digestive and Kidney Diseases, 5-alpha reductase inhibitors, like Finasteride (Proscar) and Dutasteride (Avodart), block the production of dihydrotestosterone, a male hormone that can accumulate in the prostate and cause prostate growth. The blocking of dihydrotestosterone helps shrink an enlarged prostate and can help treat UI [26].Lastly, in 2011 the FDA approved OnabotulinumtoxinA(Botox) for the treatment of urinary incontinence related to spinal cord injury, multiple sclerosis, or other nervous system disorders. These injections work by blocking the action of ACh and paralyzing the bladder muscle $[16,18]$. However, it is important to remember the side effects of these medications. Many individuals with comorbid health conditions may not be able or willing to take these drugs. For example, patients with hypertension should avoid Mirabegron because it can increase blood pressure [1].Those 
who have glaucoma may not take anticholinergic drugs due to their effect of dilation of the pupils [16]. Anticholinergics and alpha blockers may cause tachycardia; therefore, patients who suffer from cardiovascular disease should avoid these medications $[4,16]$. Furthermore, 5 -alpha reductase inhibitors may cause sexual dysfunction. Men may also experience a side of effect of impotence. Pregnant women should avoid all of these medications due to their possible effects on the fetus [34-36].

A form of treatment that is widely used in the medical field today is the use of a catheter. Catheters are thin, flexible tubes that are inserted into the bladder through the urethra to drain the bladder [26].Catheters can be inserted in the bladder through the urethra, a procedure referred to as an indwelling or Foley catheter, or an incision can be made in the abdomen where the catheter is then placed in the urethra, a suprapubic catheter. Catheters have many advantages. For example, a catheter may be used for both long-term and short-term use. Inserting a Foley catheter is not difficult once a person is trained in the procedure [28]. Ahealth care provider may teach an individual how to self- catheterize [32]. This type of catheterization is called intermittent catheterization. Having a patient insert their own catheter can help improve self-care and independence. Individuals who perform self-catheterization have a reduced risk of infections due to normal flora. Normal flora are the non-pathogenic bacterium that act as barriers to infection. Patients who perform self-catheterization have healthier flora because they do not leave their catheter in as long as an indwelling catheter, and they can regularly clean their skin when the catheter is not in place [23].

Indwelling catheters are cost effective compared to other treatments of UI. However, catheters should not be the first choice when attempting to control UI. Catheters have many risks factors. Urinary catheters can cause urinary tract infections (UTIs).In fact, catheters are the number one cause of UTIs. According to the Journal of Community Nursing, urinary catheters can also cause "urethra trauma, urethral stricture formation/perforation in addition to other complications such as encrustation, bladder calculi and neoplastic changes" [17]. In addition to these physical risk factors, urinary catheters can also lead to changes in a patient's sex life. Indwelling catheters should be removed before intimacy takes place, which may cause embarrassment [28].

Lastly, patients may always opt for surgery to correct their urinary incontinence. Injecting bulking agents into the urethra is a procedure which may be effective for both men and women. A bulking agent, usually collagen, is injected into the tissues surrounding the urethra. This helps the urethra close tightly and keeps urine from leaking out. However, this procedure is not suitable for people who are allergic to collagen [10].The two most common types of procedures for urinary incontinence in women are sling procedures and suspension procedures. According to the Mayo Clinic (2008), a sling procedure typically uses mesh to create a supportive sling under the urethra, which keeps the urethra closed. Suspension procedures require stitches to keep the bladder and urethra from sagging [20]. The most common type of surgical procedure for $\mathrm{UI}$ is the Mid urethral sling in which thin strips of mesh tape are inserted through a vaginal incision to reinforce the urethra. However, many women do not want to use mesh and often prefer the bladder neck sling because it uses the patient's own tissue to make the strong sling.

If the patient is experiencing prolapse of other pelvic organs, a surgeon may recommend Burch colposuspension. This type of surgery involves the surgeon opening the lower abdomen and placing stiches near the bladder neck, which helps keep the bladder and urethra in place [20].Men with BPH may try transurethral microwave therapy (TUMT) to treat their BPH and eliminate UI.TUMT is a minimally invasive procedure where heat is applied to a special catheter that is inserted into the urethra. Once the temperature reaches the appropriate level, the tissues within the prostate are destroyed. This relieves pressure around the urethra [15].Another type of minimally invasive procedure available tomen with $\mathrm{BPH}$ is transurethral needle ablation (TUNA). This procedure is done by putting a radiofrequency needle into the urethra. The heat from the frequency of the radio waves kills prostate tissue, relieving pressure around the urethra [13].Another surgery that men with $\mathrm{UI}$ related to BPH may chose is transurethral resection of the prostate (TURP).TURP involves inserting an endoscope and a cutting instrument into the urethra to remove portions of the prostate that may be narrowing the urethra. This is one of the most effective treatments for BPH. However, TURP is a more invasive surgery and may not be the best option for everyone due to unintended side effects such as unwanted leaking and impotence [33].

\section{Case Study: Foley Catheter Due to Urinary Incontinence}

The following presents a case study of a male client, Mr. C., who has a Foley catheter in place. A Foley catheter, also known as an indwelling catheter, is a tube that drains urine from the bladder, which can help treat urinary incontinence. It is easily inserted into the bladder through the urethra [6].A small balloon is inflated with a sterile liquid to prevent the patient from pulling it out. Timing with catheters is critical because the longer a Foley catheter is in place, the greater the chance of the patient developing a urinary tract infection (UTI).A urinary tract infection is usually caused by a bacterium that gets pushed into the bladder, a sterile environment [5].

Mr. C is a 72-year-old male who has an admitting diagnosis of a change in mental status. He has history of benign prostatic hyperplasia (BPH), an enlarged prostate. According to the Urinary Care Foundation (2017), "as the prostate enlarges, it can then squeeze down on the urethra [38]. The bladder's wall becomes thicker."This means that the bladder wall is likely to weaken, leading to the inability to completely empty the bladder, a common symptom of urinary incontinence. A Foley catheter was chosen as the best option for Mr. $\mathrm{C}$ due to urinary retention most likely caused by his history with BPH. Mr. C's history also includes recurrent UTIs, chronic kidney disease related to diabetic neuropathy, and hypertension nephrosclerosis. 
A urinalysis of Mr. C's urine was performed, which was yellow in color and cloudy in clarity. The urinalysis also gave crucial insight to Mr. C's lab values and their association with the possibility that Mr. C had developed a UTI. The two elevated lab values that were highlighted in Mr. C's urinalysis were the leukocyte and nitrite levels. Leukocyte esterase is produced by neutrophils in the body and is a normal response to pyuria, pus in the urine from an infection. Another indication of a possible infection is the elevated nitrite levels, which are not normally found in the urine. Urologists determine the presence of bacteria in the urine due to the fact that bacteria reduce urinary nitrates to nitrites [30].The relevance of Mr. C's possible UTI adds another factor that could be a reason he's experiencing urinary retention.

Mr. C has a complex case when it comes to his urinary incontinence. His Foley catheter was supposed to help his UI, but instead it may have caused complication. It cannot be determined if Mr. C already had a UTI because tests were not performed prior to the insertion of his catheter. If Mr. C already had the UTI, it is possible that catheterization was a contributing factor to his urinary incontinence and possibly his confusion $[30,3]$. Mr. C's health care provide rstreated his UTI first by prescribing antibiotics [30]. He was also prescribed a combination drug therapy of alpha blockers and 5-alpha reductase inhibitorsas described above due to his history of $\mathrm{BPH}$ for the purpose of treating his $\mathrm{UI}$ [26]. Following a negative urinalysis, Mr. C. was taught Kegel exercises to strengthen his pelvic muscles. Within three months, Mr. C was able to control his urination and return to his activities of daily living.

Conflicts of Interest: The authors confirm that there is no conflict of interest regarding this manuscript.

\section{References}

1. Astellas Pharma U S, What is myrbetriq? Inc.(2016).

2. Bardsley A. An overview of urinary incontinence. Br J Nurs. 2016; 25(18): S14-S20. doi: 10.12968/bjon.2016.25.18.s14

3. Barini-García M. Urinary incontinence fact sheet. Office of Women's Health. 2012

4. Blood Pressure Association. Non-standard medicines for high blood pressure.2008

5. Brusch, J. Catheter-related urinary tract infection. Medscape.2015.

6. Catheter-associated urinary tract infection (CAUTI) event. Deviceassociated Module.2014

7. Coyne K S, Zhou Z, Thompson C, Versi E. The impact on health-related quality of life of stress, urge and mixed urinary incontinence. BJU Int. 2003; 92(7): 731-735. doi:10.1046/j.1464-410X.2003.04463.x

8. Elliott C S, Comiter C V. The effect of angiotensin inhibition on urinary incontinence: Data from the national health and nutrition examination survey (2001-2008). Neurourol and Uro dyn, 2014; 33(8):1178-1181. doi: $10.1002 /$ nau. 22480

9. Figueroa T. Urinary incontinence in children (enuresis). Merck Manual. 2016

10. Gill, B. Injectable bulking agents for incontinence. Medscape. 2015

11. Houser E. Healthy diet, happy bladder: How diet affects bladder behavior. Cancer Connect. 2013
12. Jasmin L. Delirium. New York Times. 2013.

13. Kacker R., Williams S. Endourologic procedures for benign prostatic hyperplasia review of indications and outcomes. Urol J. 2011; 8(3): 171-176.

14. Khandelwal $C$, Kistler C. Diagnosis of urinary incontinence. Am Fam Physician, 2013; 87(8): 543-550.

15. Larson B, Mynderse L, Somers V, Jaff M, Evans Wand et al. Blood pressure surges during office-based transurethral microwave therapy for the prostate. Mayo Clin Proc. 2008; 83(3):309-312.doi: 10.4065/83.3.309.

16. Drugs and supplements: anticholinergics and antispasmodics (oral route, parenteral route, rectal route, transdermal route). Mayo Foundation for Medical Education and Research. 2017

17. Kegel exercises: A how-to guide for women. Mayo Foundation for Medical Education and Research. 2015

18. Bladder control problems: Medications for treating urinary incontinence. Mayo Foundation for Medical Education and Research. 2014a

19. Diseases and conditions: urinary incontinence. Mayo Foundation for Medical Education and Research.2014b.

20. Mayo Foundation for Medical Education and Research. Stress incontinence: Surgical treatments for women. Mayo Clin Health Letter: Tools for Healthier Lives, 2008; 26(8): 1- 8.

21. Musa M U. The role of urine investigations in urology practice. Open J Orthop. 2015; 5:90-99. doi:10.4236/ojo.2015.54012

22. Study shows benefits, drawbacks, for women's incontinence treatments. National Institutes of Health. 2012

23. Newman $D$, Willson $M$. Review of intermittent catheterization and current best practices. Urol Nurs. 2011; 32(1): 12-28.

24. Urinary incontinence - causes. NHS (2016).

25. Bladder control problems in women (urinary incontinence). National Institute of Diabetes and Digestive and Kidney Diseases. (2016).

26. Bladder control problems in men (urinary incontinence). National Institute of Diabetes and Digestive and Kidney Diseases. 2015.

27. Urinary incontinence in women statistics. Phoenix Physical Therapy, PLC. 2017.

28. Pomfret, I. Indwelling urinary catheters: friend or foe?. I Community Nurs. 2008; 22(7): 17-19.

29. Schmiemann G, Kniehl E, Gebhardt K, Matejczyk M, \& et al. The diagnosis of urinary tract infection: A systematic review. Dtsch Arztebl Int. 2010; 107(21): 361-367. doi: 10.3238/arztebl.2010.0361

30. Simmerville J, Maxted W, Pahira J. Urinalysis: A comprehensive review. Am Fam Physician. 2005; 71(6): 1153-1162.

31. Smith M, Hussain M, Seth J, Kazkaz H, Panicker J. Stress urinary incontinence as the presenting complaint of benign joint hypermobility syndrome. $J$ Royal Society Med. 2012; 3(9): 66. doi:10.1258/shorts.2012.012005.

32. Sobol J. Urinary incontinence. Med line Plus. NIH U.S. National Library of Medicine. 2014

33. Thiruchelvam $N$. Surgical therapy for benign prostatic hypertrophy/bladder outflow obstruction. Indian J Urol. 2014; 30(2): 202-207. doi:10.4103/09701591.126907

34. Trost $L$, Saitz $T$ R., Hellstrom WJ. Side effects of 5-alpha reductase inhibitors: A comprehensive review. Sex Med Rev. 2013; 2(1):24-41. doi:10.1002/smrj.3.

35. Biofeedback for incontinence. UCSF Medical Center. 2017a.

36. Bladder training. UCSF Medical Center. 2017b.

37. Urinary incontinence. University of Maryland Medical Center. 2012.

38. What is benign prostatic hyperplasia (BPH)?. Urology Care Foundation. 2017.

39. Taking medicine during pregnancy. WebMD. 2017. 